Research paper

\title{
Cyclodextrins enable indirect ultrasensitive Raman detection of polychlorinated biphenyls captured by plasmonic bubbles
}

\author{
Irene Vassalini ${ }^{\text {a,c, e }}$, Nicolò Bontempi ${ }^{\text {a }}$, Stefania Federici ${ }^{\mathrm{a}, \mathrm{b}}$, Matteo Ferroni ${ }^{\mathrm{c}, \mathrm{f}}$, \\ Alessandra Gianoncelli $^{\text {a,d }}{ }^{\text {, Ivano Alessandri }}{ }^{\text {a,c,e,* }}$ \\ ${ }^{\text {a } I N S T M, ~ U d R ~ B r e s c i a, ~ I t a l y ~}$ \\ ${ }^{\mathrm{b}}$ Chemistry for Technologies Laboratory, Mechanical and Industrial Engineering Department (DIMI), University of Brescia, via Branze 38 , 25123 Brescia, Italy \\ ${ }^{\mathrm{c}}$ Department of Information Engineering (DII), University of Brescia, via Branze 38, 25123 Brescia, Italy \\ ${ }^{\mathrm{d}}$ Department of Molecular and Translational Medicine, University of Brescia, Viale Europa 11, 25123, Brescia, Italy \\ e INO-CNR, via Branze 38, 25123 Brescia, Italy \\ ${ }^{\mathrm{f}}$ IMM-CNR, via Gobetti 101, 40129 Bologna, Italy
}

\section{A R T I C L E I N F O}

\section{Keywords:}

SERS

Persistent organic pollutants (POPs)

Polychlorinated biphenyls (PCBs)

Ultrasensitive detection

Preconcentration

Alginate hydrogels

Silver nanoparticles

$\beta$-cyclodextrin

\begin{abstract}
A B S T R A C T
Capture, detection and removal of persistent organic pollutants (POPs) from water, air and soil are among the hot topics of research in environmental science.

Here we report the first example of indirect ultrasensitive detection of polychlorinated biphenyls in water, obtained by analysing the Raman signals of silver-cyclodextrin supracrystals resulting from drying-induced aggregation of plasmonic alginate bubbles. Principal component analysis allowed to extend the detection limit to the level of $1 \mathrm{ng} / \mathrm{L}$, which is two orders of magnitude lower than that requested by current legislation.
\end{abstract}

The presence of persistent organic pollutants (POPs) in water, air and soil has a dramatic impact on ecosystems and human health [1]. Raman spectroscopy has been extensively investigated as an alternative or complementary tool to conventional, yet cumbersome, laboratory techniques, such as chromatography and mass spectrometry, as it provides the chemical fingerprint of POPs and can be easily implemented to the analysis of aqueous solutions because, unlike infrared spectroscopy, it is almost insensitive to water [2,3]. However, trace-level Raman detection of these types of molecular targets in aqueous matrices is usually hampered by their low Raman cross-section. The use of plasmonic metal nanoparticles allowed to exploit Surface Enhanced Raman Scattering (SERS) and extend the analytical capability of Raman-based assays to the level of single molecule, yet these impressive results have been reported only for organic dyes characterized by high Raman cross-section and optical absorption in resonance with the Raman laser source $[4,5]$.

Novel strategies based on pre-concentrating the analytical target on SERS-active omniphobic substrates have been recently developed, in order to further increase sensitivity [6-8]. These methods are very promising for ex-situ Raman sensing. However, the development of reliable probes capable of a simultaneous label-free detection and efficient removal of POPs under real working conditions is still an urgent and unmet goal [9]. We recently demonstrated that alginate-based hydrogels loaded with $\beta$-cyclodextrin-capped silver nanoparticles (Ag@ $\beta$-CD NPs) are efficient tools for encapsulation, detection and removal of different types of organic pollutants, including dyes and pharmaceutical compounds, without need of any microfluidic chip or superhydrophobic platforms [10]. The working principle of these plasmonic bubbles consists in the synergistic interaction of multiple components (Fig. 1). The silver nanoparticles are utilized for amplifying Raman scattering, while $\beta-\mathrm{CD}$ as silver nanoparticle stabilizers and molecular pan-specific receptors for a large variety of pollutants. Finally, alginate bubbles are environment-friendly, dispersible hydrogel capsules for encapsulating the SERS-active receptors and further capturing/preconcentrating the pollutant targets.

Here we explore the possibility to extend the application of these type of plasmonic bubbles to other classes of POPs, through a novel, radically different approach based on indirect Raman analysis.

In this work, the molecular targets are represented by polychlorinated biphenyls (PCBs), which have been recognized as very

\footnotetext{
* Corresponding author.
} 
a) Components

\section{b) Capture/Detection Scheme}

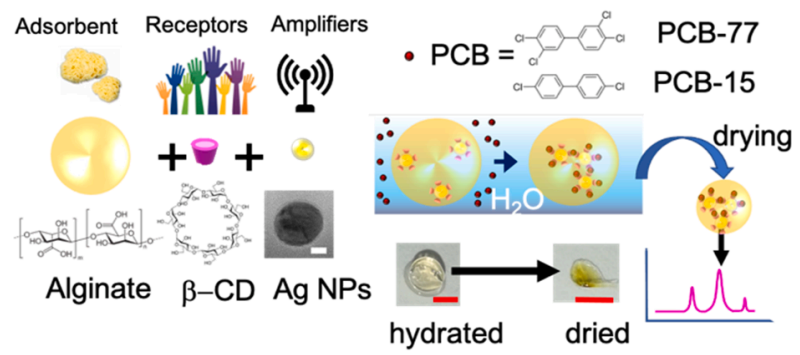

Raman analysis

Fig. 1. A) Individual building blocks utilized to fabricate the plasmonic bubbles: adsorbent (alginate hydrogel bubbles), PCB-receptors ( $\beta$-CD) and optical antennas for amplification of the Raman signal (Ag NPs, scale bar: $5 \mathrm{~nm}$ ); b) scheme of capture and detection of PCBs. The optical images of plasmonic bubbles in hydrated and dried form are shown (Scale bars: $1 \mathrm{~mm}$ ).

dangerous carcinogenic agents and endocrine disruptors even at level of traces [11] and are among the most challenging types of POPs in terms of detection. Due to the low solubility of PCBs in water, the possibility to achieve reliable detection at low concentration is very important. In drinking water, the maximum level of PCBs allowed by the U.S. Environmental Protection Agency (EPA) is $0.5 \mathrm{mg} / \mathrm{L}$ [12].

High sensitivity in PCB detection, up to $10^{-6}-10^{-7} \mathrm{M}$, was reported for conventional plasmonic SERS substrates in the absence of receptors [13-17]. However these approaches can be utilized only in lab-scale experiments as they do not allow for a direct capture of PCBs in water under real world conditions. For example, Bantz and Haynes demonstrated the detection of PCBs by partition layer-modified SERS substrates [18]. This method relies on the spectral subtraction of signals of decanethiols utilized for coating Ag NPs before and after addition of PCB solutions, which exposes it to pitfalls due to the presence of other possible apolar interfering species.

Affinity agents can help addressing these issues. $\beta$-CDs have been widely utilized as abiotic receptors to bind a large variety of molecular guests, by taking advantage of their hydrophobic inner cavity, which makes them suitable to host any apolar moiety (e.g. phenyl groups, hydrophobic chains...) of a given molecular guest with a size compatible with that of the cavity $(0.65 \mathrm{~nm})$ [19]. For this reason, $\beta$-CDs have been successfully utilized as receptors for PCBs [10]. Depending on matching between the size of the $\beta$-CD cavity and that of a given PCB, the hostguest complexation energy can be slightly different, ranging from 20 to $40 \mathrm{Kcal} \mathrm{mol}^{-1}$ in water solutions [20].

However, the SERS sensitivity of $\beta$-CD-functionalized plasmonic substrates towards PCBs has been limited to $10^{-4}-10^{-5} \mathrm{M}[21,22]$.

Here we aim at exploiting the pivotal role of $\beta$-CDs in inducing selfassembly of silver nanoparticles in response to analyte-receptor binding events, to create Ag@ $\beta$-CD supracrystals and aggregates able to keep the information of the presence of PCBs. $\beta$-CD-mediated self-assembly has been already utilized for controlling nanoparticle aggregation and for UV-Vis detection of analytes [23] but not for Raman sensing. In fact, most of the organic molecules, including PCBs, exhibit Raman signals that fall in the same spectral region $\left(400-1800 \mathrm{~cm}^{-1}\right)$ of Raman modes originated from intermolecular interactions among the metal@ $\beta$-CD nanoparticles. Thus, at low concentration, the spectral features of the analyte are completely hidden by the Raman modes of the hybrid aggregates [21]. However, these Raman modes could be influenced by the presence of analytes and give rise to spectra that can be utilized for indirect sensing. We tested our hypothesis through preliminary experiments that compared the Raman spectra of tetrachlorobiphenyl (PCB77) captured by plasmonic bubbles with or without $\beta$-CD receptors. Both types of bubbles were extracted from water contaminated by PCBs and dried at room temperature. All experimental details are reported in ESI 1. Upon drying, the bubbles shrink up to $\sim 70 \%$ and their average

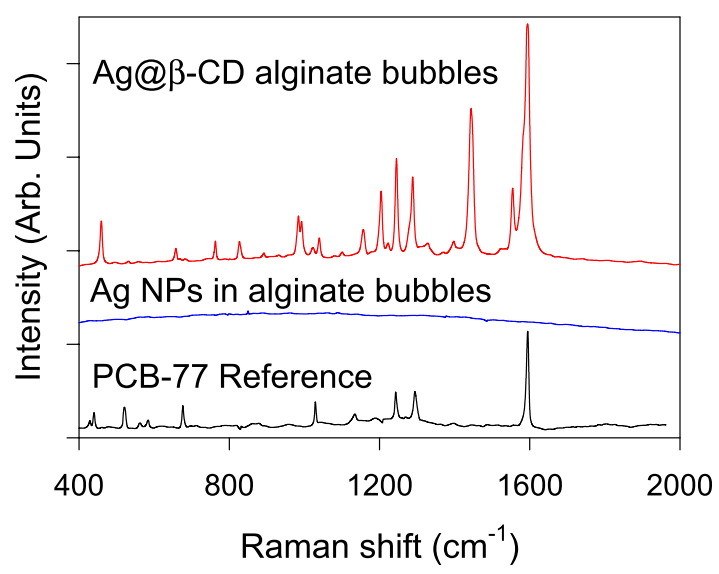

Fig. 2. Raman detection and analysis of the captured PCBs. Raman spectra of PCB-77 adsorbed from an aqueous solution at the initial concentration of $3.4 \times$ $10^{-4} \mathrm{M}$ by either Ag@ $\beta$-CD or $\beta$-CD-free plasmonic alginate dried bubbles. The reference spectrum is also shown for comparison.

weight is reduced by two orders of magnitude, from about 10 to $0.1 \mathrm{mg}$, which means that water contribute by $99 \%$ to the overall mass of the original bubble. As a result of drying, the silver nanoparticles are induced to aggregate.

Fig. 2 shows the Raman spectra of PCB-77 captured by soaking the plasmonic bubbles for $16 \mathrm{~h}$ in polluted aqueous solutions at a nominal concentration of $3.4 \times 10^{-4} \mathrm{M}$, corresponding to $100 \mathrm{mg} / \mathrm{L}$. The Raman spectra acquired from analogous bubbles made with naked Ag NPs (i.e. in the absence of $\beta-\mathrm{CD}$ receptors) do not show any signal associated to the presence of the PCB. On the other hand, the Raman spectra acquired from the Ag@ $\beta-C D$ alginate bubbles contain the main spectral features of the pollutant, such as the stretching peaks of both the biphenyl rings around $1600 \mathrm{~cm}^{-1}$, the stretching on bridge at $1294 \mathrm{~cm}^{-1}, \mathrm{C}-\mathrm{H}$ wagging at $1240 \mathrm{~cm}^{-1}$, trigonal stretching ring at $1028 \mathrm{~cm}^{-1}$ and C-Cl stretching and deformation ring modes at 1134 and $674 \mathrm{~cm}^{-1}$. In comparison to the reference spectrum, we also observe the signal of the $\beta$-CD receptors [10], as well as extra Raman modes (see, for example the peak splitting in the $1570-1600 \mathrm{~cm}^{-1}$ region) that suggest the formation of $\beta$-CD:PCB host-guest complex aggregates.

As shown in ESI 2, both types of plasmonic bubbles (with or without $\beta$-CD) exhibit a comparable adsorption capability. The slight increase in PCB absorption observed in $\beta$-CD-functionalized bubbles is not sufficient to justify such a remarkable difference in Raman response, which might originate from the key role of $\beta-\mathrm{CD}$ in driving the formation of silver aggregates with an efficient distribution of the plasmonic hotspots. The fact that these signals were only observed in this concentration range, which corresponds to a nearly-stoichiometric ratio between host and guest molecules, confirms previous computational studies on the formation of $\beta$-CD-PCB complexes [20].

On the other hand, higher concentration $\left(3.4 \times 10^{-3} \mathrm{M}\right)$ of the analyte did not result in any significant enhancement of the Raman spectra, which are similar to that of the PCB-77 reference solutions (ESI 3-1). This is due to the fact that SERS is a surface effect, which is maximized only when the analytical targets are in close proximity to the plasmonic metal nanoparticles and typically vanishes out for distances exceeding 5-10 $\mathrm{nm}$. As expected, at low concentration $\left(<3.4 \times 10^{-5} \mathrm{M}\right)$, direct detection of the PCB Raman modes is hampered by the high density of Raman modes resulting from aggregated Ag@ $\beta$-CD nanoparticles, which are not observed in either $\beta$-CD- free or pure alginate bubbles (Fig. 3a). The strong influence of non-covalent interactions on the Raman spectra of organic and hybrid organic-inorganic crystals has been extensively investigated in previous studies [24]. In particular, Loftsson and co-workers and Baglioni and co-workers elucidated the complexity of $\beta-\mathrm{CD}$ crystallization [25,26]. For sensing purposes, it would be important to evaluate whether these aggregates can be 


\section{a) Drying without PCBs}

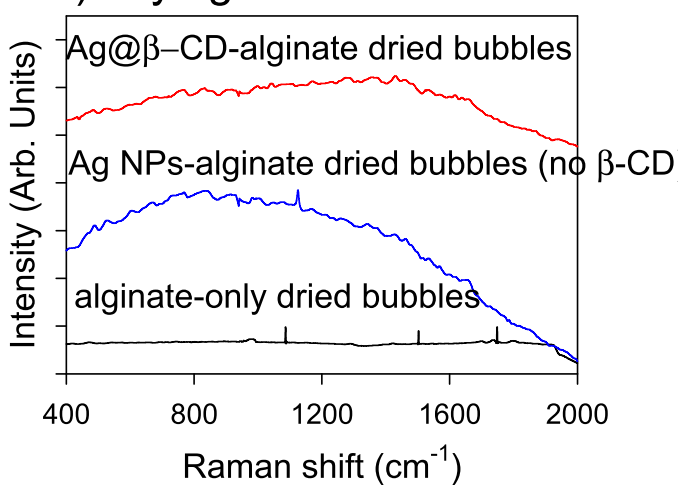

b) Drying with PCBs

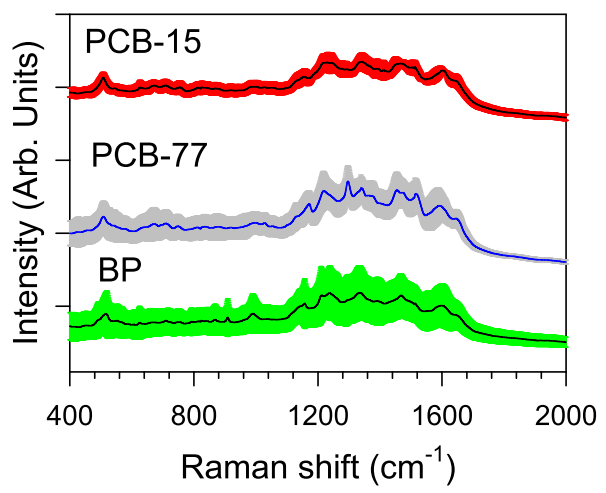

Fig. 3. a) Examples of Raman spectra of pure alginate, and b-CD-free alginate and Ag@ $\beta$-CD alginate dried bubbles obtained from water without PCBs. b) Examples of Raman spectra obtained from Ag@ $\beta$-CD alginate bubbles that have been extracted and dried from water containing BP, PCB-77 and PCB-15. The plasmonic bubbles were soaked for 2 weeks in PCB solutions $(10 \mu \mathrm{g}$ / L). The continuous line represents the averaged spectrum, the shaded contour is formed by standard deviation of the spectra (an enlarged view is shown in Fig. ESI 3.4). a)

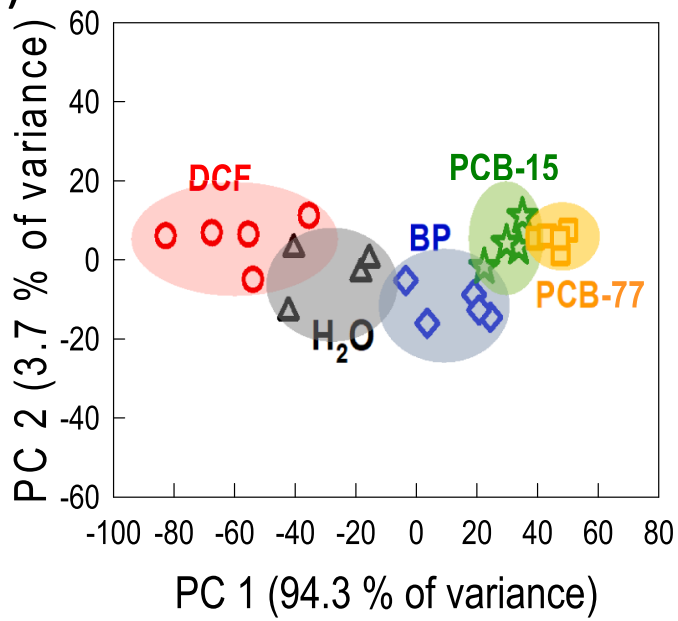

c)

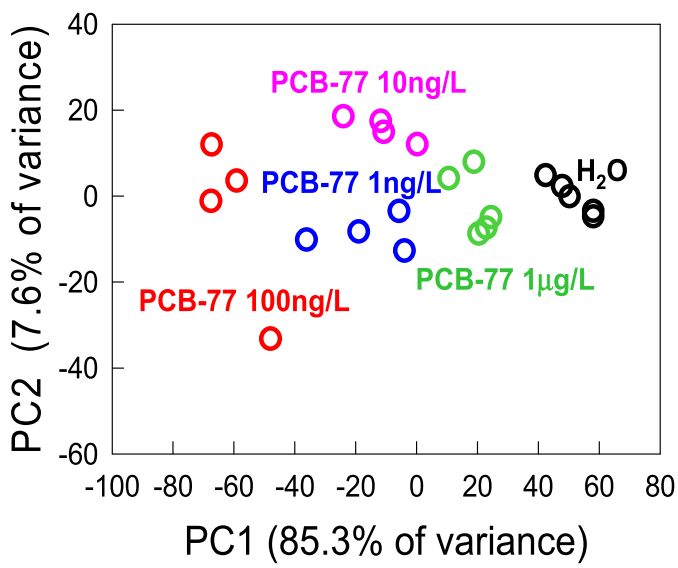

b)

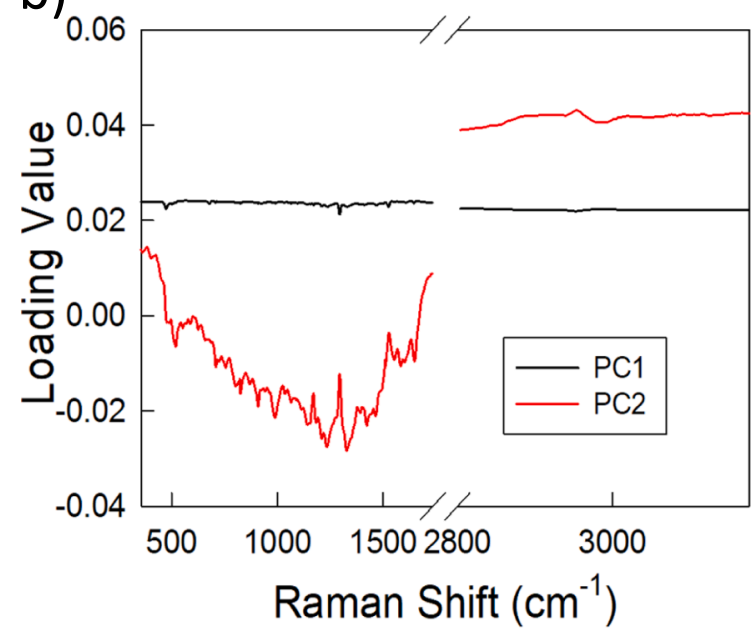

d)

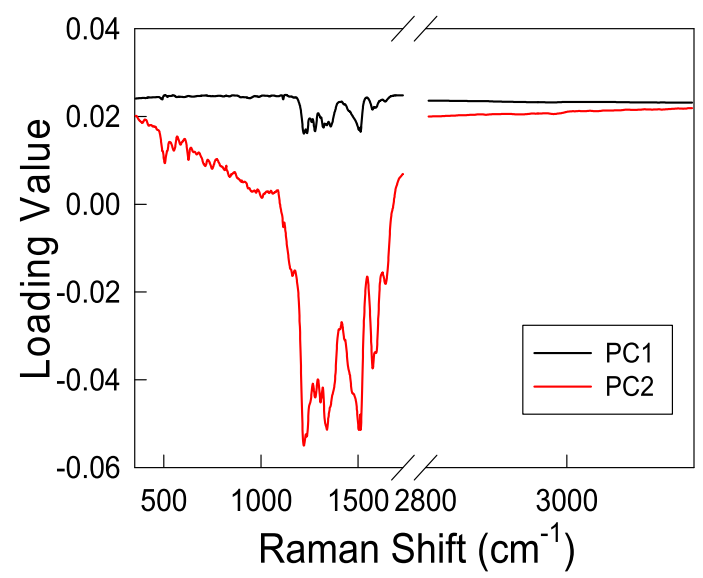

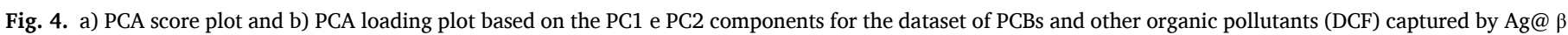

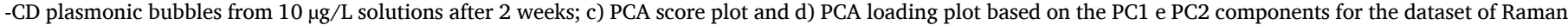
spectra of PCB-77 solutions at different concentration. Pure water is also reported for reference. The limit of detection is extended to 1 ng/L.

distinguished on the basis of the analytes that have or have not been captured and distinguish among different types of PCBs and between PCBs and other types of compounds captured by $\beta$-CD receptors. In order to investigate these points, in addition to PCB-77, other two different types of biphenyl compounds, i.e. non-chlorinated biphenyl (BP) and 1,4 dichlorobiphenyl (PCB-15), were analysed using the same procedure. Fig. 3b shows examples of Raman spectra of the three different biphenyl compounds that have been captured by plasmonic bubbles after the same soaking time (two weeks) and at the same nominal concentration of the PCB-contaminated solution (10 mg/L). For simplicity, we report the averaged Raman spectra of the PCBs. The standard deviation is indicated by the shaded contour of the averaged 
spectrum. At least 30 measurements per specimen have been carried at different soaking time (16 h, 4 days, 1, 2, 3 and 4 weeks). In general, at any given soaking time the spectra of the different biphenyl compounds exhibit the Raman modes of $\beta$-CDs, with variations of intensity and positions that reflect their local order of aggregation.

A direct comparison of Fig. 3 a and $b$ shows that the Raman spectra of Ag@ $\beta$-CD aggregates either in the presence or absence of PCBs are significantly different. On the other hand, the spectra of Ag@ $\beta$-CD aggregates soaked in solutions with different types of PCBs are quite similar and their distinction is not trivial, as the PCB-specific Raman modes are not detectable and information about their presence and identity is convoluted in the signals of the $\beta$-CD aggregates. For this reason, in order to assess the applicability of plasmonic bubbles for a fast and reliable detection of PCBs and, in general, organic pollutants, at very low concentration, we processed the Raman data through principal component analysis (PCA). PCA is a well-known chemometric technique utilized to extract few variables from a high dimensional data set and visualize them in a lower dimensional space of uncorrelated variables, which are linear combinations of the original ones, the so-called Principal Components (PCs) [27]. This type of multivariate analysis is particularly useful to disentangle the most significant information contained in the spectra. Fig. 4 a shows the results of PCA on Raman spectra acquired from Ag@ $\beta$-CD plasmonic bubbles that have been soaked in solutions containing PCB-15, PCB-77, BP and diclofenac (DCF) in the low concentration range, as well as in non-contaminated mineral water. PCA operates a clear distinction among the samples. In particular, DCF is clearly separated from biphenyl compounds, which are in turn distinguished on the basis of the number of chlorine atoms. Moreover, both types of POPs (biphenyls and diclofenac) are distinguished from mineral water. This approach allows to take advantage of indirect information from the Raman spectra of $\mathrm{Ag} @ \beta$-CD nanoparticle aggregates to rapidly detect the presence of different POPs. Fig. $4 \mathrm{~b}$ shows the evaluation of the detection limit for PCB through PCA in a low concentration range (1 $\mathrm{mg}-1 \mathrm{ng} / \mathrm{L})$. PCA enabled to sort data in distinguishable groups. This strategy allows for a reliable detection of PCBs from aqueous solutions in the order of $1 \mathrm{ng} / \mathrm{L}$, a concentration that is two orders of magnitude lower than the EPA limit $(0.5 \mathrm{mg} / \mathrm{L})$ [12].

In summary, this study shows that the Raman modes of $\beta-\mathrm{CD}$ receptors coupled to Ag NPs in dried plasmonic bubbles containing low concentration (from $\mathrm{mg} / \mathrm{L}$ to $\mathrm{ng} / \mathrm{L}$ ) of PCBs can be differentiated from those of reference, pollutant-free bubbles. Moreover, different types of PCBs can be distinguished on the basis of PCA analysis. Further differentiations between the Raman spectra of PCB-loaded and bubbles carrying pharmaceutical compounds were also assessed. These experiments suggest that, at low concentration, all information about the identity of different analytes is encoded in Raman spectra of the Ag@ $\beta$-CD aggregates and can be revealed through this indirect approach. The origin of the different, non-covalent interactions between the analytes and Ag@ $\beta$-CD nanoparticles, which occur during complexation and drying, is still unclear and deserves further investigation. These studies are challenging and could include the use of cryo-TEM and synchrotron radiation techniques to characterize the hybrid aggregates. In fact, conventional TEM analysis does not allow to include alginate bubbles and gives rise to artifacts.

A hypothesis is that small amounts of PCBs might be sufficient to reduce the number of water molecules associated with the $\beta$-CD receptors, with direct consequences on the non-covalent interactions that drive the drying-induced aggregation of $\mathrm{Ag} @ \beta$-CD nanoparticles.

However, from a sensing viewpoint, a full understanding of the mechanism of aggregation is not mandatory at this stage of the research, as the significance of the experiments has been assessed by PCA, which enabled to manage the complexity and variability of Raman data at low concentration ranges, sorting out similar spectra originated from different analytes. Notably, in the case of PCBs investigated in the present work, the limit of detection has been lowered by two orders of magnitude with reference to the current legislation limits. The concept of indirect Raman detection is quite general and versatile. It does not need of using any dyes, labels or Raman reporters and could be extended and suitably adapted to the detection of many other target species by means of novel strategies of sensing that have been recently developed [28-33]. Moreover, it offers a large variety of applications that are not limited to environmental analysis and remediation, but can be extended to forensics, medicine and, in general, molecular diagnostics.

\section{Declaration of Competing Interest}

The authors declare that they have no known competing financial interests or personal relationships that could have appeared to influence the work reported in this paper.

\section{Acknowledgments}

This work was partially funded by INSTM and Regione Lombardia (Project grant: "Microsfere adattative per il monitoraggio e l'abbattimento di inquinanti persistenti-MI ADATTI E L'ABBATTI"), Italian Ministry of Agriculture Food and Forestry Policies (MIPAAF, project grant: "RESTART") and Italian Ministry of University and Research (MUR) through the PRIN project NOMEN (2017MP7F8F).

\section{Appendix A. Supplementary data}

Supplementary data to this article can be found online at https://doi. org/10.1016/j.cplett.2021.138674.

\section{References}

[1] United Nation, Final Act of the Conference of Plenipotentiaries on the Stockholm Convention on Persistent Organic Pollutant, 2001. http://www.pops.int/TheConve ntion/Overview/TextoftheConvention/tabid/2232/Default.aspx.

[2] N. Bontempi, I. Vassalini, I. Alessandri, All-dielectric core/shell resonators: From plasmon-free SERS to multimodal analysis, J. Raman Spectrosc. 49 (2018) 943-953, https://doi.org/10.1002/jrs.5330.

[3] I. Alessandri, J.R. Lombardi, Editorial: Surface Enhanced Raman Scattering: New Theoretical Approaches, Materials and Strategies, Front. Chem. 8 (2020) 8-10, https://doi.org/10.3389/fchem.2020.00063.

[4] K. Kneipp, Y. Wang, H. Kneipp, L.T. Perelman, I. Itzkan, R.R. Dasari, M.S. Feld, Single Molecule Detection Using Surface-Enhanced Raman Scattering (SERS), Phys. Rev. Lett. 78 (1997) 1667-1670, https://doi.org/10.1103/PhysRevLett.78.1667.

[5] S. Nie, S.R. Emory, Probing single molecules and single nanoparticles by surfaceenhanced Raman scattering, Science 275 (1997) 1102-1106, https://doi.org/ 10.1126/science.275.5303.1102.

[6] I. Alessandri, L.E. Depero, Metal oxide microrings with femtoliter capacity for raman microspectroscopy, ACS Appl. Mater. Interfaces 2 (2010) 594-602, https:// doi.org/10.1021/am900904k.

[7] S. Yang, X. Dai, B.B. Stogin, T.S. Wong, Ultrasensitive surface-enhanced Raman scattering detection in common fluids, Proc. Natl. Acad. Sci. U. S. A. 113 (2016) 268-273, https://doi.org/10.1073/pnas.1518980113.

[8] M. Li, B. Dyett, H. Yu, V. Bansal, X. Zhang, Functional Femtoliter Droplets for Ultrafast Nanoextraction and Supersensitive Online Microanalysis, Small 15 (2019) 1-8, https://doi.org/10.1002/smll.201804683.

[9] P.J. Vikesland, Nanosensors for water quality monitoring, Nat. Nanotechnol. 13 (2018) 651-660, https://doi.org/10.1038/s41565-018-0209-9.

[10] I. Vassalini, G. Ribaudo, A. Gianoncelli, M.F. Casula, I. Alessandri, Plasmonic hydrogels for capture, detection and removal of organic pollutants, Environ. Sci. Nano. 7 (2020) 3888-3900, https://doi.org/10.1039/d0en00990c.

[11] IARC, Polychlorinated Biphenyls and Polybrominated Biphenyls, IARC Monogr. Eval. Carcinog. Risks Humans 107 (2016) 9-500.

[12] US Environmental Protection Agency, Drinking Water Contaminants-Standards and Regulations (US Environmental Protection Agency, Washington, DC) www. epa.gov/dwstandardsregulations.

[13] A. Alsbaiee, B.J. Smith, L. Xiao, Y. Ling, D.E. Helbling, W.R. Dichtel, Rapid removal of organic micropollutants from water by a porous $\beta$-cyclodextrin polymer, Nature. 529 (2016) 190-194, https://doi.org/10.1038/nature16185.

[14] D. Arockia Jency, M. Umadevi, G.V. Sathe, SERS detection of polychlorinated biphenyls using $\beta$-cyclodextrin functionalized gold nanoparticles on agriculture land soil, J. Raman Spectrosc. 46 (2015) 377-383, https://doi.org/10.1002/ jrs. 4654 .

[15] Y. Lu, G. Yao, K. Sun, Q. Huang, $\beta$-cyclodextrin coated SiO2@Au@Ag core/shell nanoparticles for SERS detection of PCBs, Phys. Chem. Chem. Phys. 17 (2015) 21149-21156, https://doi.org/10.1039/b000000x.

[16] S. Kawano, T. Kida, K. Miyawaki, Y. Noguchi, E. Kato, T. Nakano, M. Akashi, Cyclodextrin polymers as highly effective adsorbents for removal and recovery of 
polychlorobiphenyl (PCB) contaminants in insulating oil, Environ. Sci. Technol. 48 (2014) 8094-8100, https://doi.org/10.1021/es501243v.

[17] X. Chen, S.G. Parker, G. Zou, W. Su, Q. Zhang, B-Cyclodextrin-Functionalized Silver Nanoparticles for the Naked Eye Detection of Aromatic Isomers, ACS Nano 4 (2010) 6387-6394, https://doi.org/10.1021/nn1016605.

[18] K.C. Bantz, C.L. Haynes, Surface-enhanced Raman scattering detection and discrimination of polychlorinated biphenyls, Vib. Spectrosc. 50 (2009) 29-35, https://doi.org/10.1016/j.vibspec.2008.07.006.

[19] J. Szejtli, Introduction and general overview of cyclodextrin chemistry, Chem. Rev. 98 (1998) 1743-1753, https://doi.org/10.1021/cr970022c.

[20] P. Liu, D. Zhang, J. Zhan, Investigation on the inclusions of PCB52 with cyclodextrins by performing DFT calculations and molecular dynamics simulations, J. Phys. Chem. A 114 (2010) 13122-13128, https://doi.org/10.1021/jp109306v.

[21] J. Yuan, Y. Lai, J. Duan, Q. Zhao, J. Zhan, Synthesis of a $\beta$-cyclodextrin-modified Ag film by the galvanic displacement on copper foil for SERS detection of PCBs, J. Colloid Interface Sci. 365 (2012) 122-126, https://doi.org/10.1016/j. jcis.2011.08.075.

[22] A. Arrais, P. Savarino, Raman spectroscopy is a convenient technique for the efficient evaluation of cyclodextrin inclusion molecular complexes of azo-dye colorants and largely polarisable guest molecules, J. Incl. Phenom. Macrocycl. Chem. 64 (2009) 73-81, https://doi.org/10.1007/s10847-009-9538-5.

[23] Y. Zhao, Y. Huang, H. Zhu, Q. Zhu, Y. Xia, Three-in-One: Sensing, Self-Assembly, and Cascade Catalysis of Cyclodextrin Modified Gold Nanoparticles, J. Am. Chem. Soc. 138 (2016) 16645-16654, https://doi.org/10.1021/jacs.6b07590.

[24] I. Alessandri, L.E. Depero, All-oxide raman-active traps for light and matter: Probing redox homeostasis model reactions in aqueous environment, Small 10 (2014) 1294-1298, https://doi.org/10.1002/smll.201303166.
[25] M. Messner, S.V. Kurkov, P. Jansook, T. Loftsson, Self-assembled cyclodextrin aggregates and nanoparticles, Int. J. Pharm. 387 (2010) 199-208, https://doi.org/ 10.1016/j.ijpharm.2009.11.035.

[26] M. Bonini, S. Rossi, G. Karlsson, M. Almgren, P. Lo Nostro, P. Baglioni, Selfassembly of $\beta$-cyclodextrin in water. Part 1: Cryo-TEM and dynamic and static light scattering, Langmuir 22 (2006) 1478-1484, https://doi.org/10.1021/la052878f.

[27] S. Wold, K. Esbensen, P. Geladi, Principal Component Analysis, Chemom. Intell. Lab. Syst. 2 (1987) 37-52, https://doi.org/10.1016/0169-7439(87)80084-9.

[28] I. Alessandri, J.R. Lombardi, Enhanced Raman Scattering with Dielectrics, Chem. Rev. 116 (2016) 14921-14981, https://doi.org/10.1021/acs.chemrev.6b00365.

[29] I. Alessandri, N. Bontempi, L.E. Depero, Colloidal lenses as universal Raman scattering enhancers, RSC Adv. 4 (2014) 38152-38158, https://doi.org/10.1039/ c4ra07198k.

[30] I. Alessandri, I. Vassalini, M. Bertuzzi, N. Bontempi, M. Memo, A. Gianoncelli, "RaMassays": Synergistic Enhancement of Plasmon-Free Raman Scattering and Mass Spectrometry for Multimodal Analysis of Small Molecules, Sci. Rep. 6 (2016) 1-8, https://doi.org/10.1038/srep34521.

[31] N. Bontempi, I. Vassalini, S. Danesi, I. Alessandri, ZORRO: Zirconium oxide resonators for all-in-one Raman and whispering-gallery-mode optical sensing, Chem. Commun. 53 (2017) 10382-10385, https://doi.org/10.1039/c7cc06357a.

[32] I. Vassalini, J. Gjipalaj, S. Crespi, A. Gianoncelli, M. Mella, M. Ferroni, I. Alessandri, Alginate-Derived Active Blend Enhances Adsorption and Photocatalytic Removal of Organic Pollutants in Water, Adv. Sustain. Syst. 4 (2020) 1-11, https://doi.org/10.1002/adsu.201900112.

[33] A. Mariño-Lopez, A. Sousa-Castillo, M. Blanco-Formoso, L.N. Furini, L. RodríguezLorenzo, N. Pazos-Perez, L. Guerrini, M. Pérez-Lorenzo, M.A. Correa-Duarte, R. A. Alvarez-Puebla, Microporous Plasmonic Capsules as Stable Molecular Sieves for Direct SERS Quantification of Small Pollutants in Natural Waters, ChemNanoMat. 5 (2019) 46-50, https://doi.org/10.1002/cnma.201800355. 